\title{
AN EXTENSION OF THE INTERPOLATION THEOREM OF MARCINKIEWICZ II
}

\author{
SATORU IGARI*)
}

(Received July 30, 1963)

1. Introduction. The purpose of this paper is to give the detailed proof of the theorem which is announced in my previous paper [2], that is, to show that Marcinkiewicz's theorem on the interpolation of operators (e.g.see A.Zygmund [6; Chap. XII]) holds good for Hardy class $H_{p}(p \geqq 1)$.

$H_{p}$-class $(\mathrm{p}>0)$ is the space of all functions analytic in the unit circle such that

$$
\|\varphi\|_{p}=\lim _{r \rightarrow 1-0}\left\{\frac{1}{2 \pi} \int_{-\pi}^{\pi}\left|\varphi\left(r e^{i \theta}\right)\right|^{p} d \theta\right\}^{1 / p}
$$

is finite. For $p \geqq 1$ this class is equivalent to the space of functions in $L_{p}(-\pi, \pi)$ with the ordinary $L_{p}$-norm such that their Fourier expansion is power series type, that is,

$$
\sum_{n=0}^{\infty} a_{n} e^{i n \cdot x}
$$

Our method of proof depends on the real one and can be applied to some $n$-dimensional analogues of $H_{p}$-class.

Sections 2 and 3 contain the case of one variable $H_{p}$-space.

Sections 4 and 5 treat $n$-dimensional analogues of $H_{p}$-space.

Section 6 contains some applications to the theorems on Fourier series.

2. Two Lemmas. We begin by defining some notations. Let $f \in L_{p}(-\pi, \pi)(p>1)$ be periodic with period $2 \pi$ and its Fourier expansion be

$$
f(x) \sim \sum_{n=-\infty}^{\infty} a_{n} e^{i n x},
$$

then its conjugate function $\widetilde{f}(x)$ is defined by

$$
\widetilde{f}(x)=\lim _{\varepsilon \rightarrow 0} \frac{1}{\pi} \int_{\pi \geqq|y| \geq \varepsilon} \frac{f(y)}{2 \tan (x-y) / 2} d y
$$

or equivalentely

$$
\widetilde{f}(x) \sim-\sum_{n=-\infty}^{\infty} i(\operatorname{sign} n) a_{n} e^{i n x} .
$$

*)The author thanks Professors G. Sunouchi and S. Yano for their encouragement and valuable suggestions. 
Therefore if we put

$$
K f=(f+i \widetilde{f}) / 2
$$

for $f \in L_{p}(-\pi, \pi)(p>1)$, then by expressions (1.1) and (2.2), we have $K f \in H_{p}$ and in particular if $f \in H_{p}(p \geqq 1)$, then we have $K f=f$.

Our first lemma is a modification of that of L.Hörmander's [1; p. 115].

LEMMA 1. Let $f \in L_{p}(-\pi, \pi)(\infty>p \geqq 1), p \geqq r \geqq 1$ and let us define $a_{0}$ by

$$
\frac{\pi}{2}=\frac{1}{a_{0}^{r}} \int_{-\pi}^{\pi}|f(x)|^{r} d x,
$$

then for every $a>a_{0}$, the following decomposition of $f$ is possible;

(i) $f=u+u^{\prime}, u^{\prime}=v+w, w=\sum_{k=1}^{\infty} w_{k}$,

(ii) $\quad u=f$, if $|f|<a$ and $u=0$ elswhere,

(iii) $\quad|v(x)| \leqq 2^{1 / r} a$ for a.e. $x$ in $(-\pi, \pi)$,

$$
\int_{-\pi}^{\pi}|v(x)|^{s} d x \leqq \int_{-\pi}^{\pi}\left|u^{\prime}(x)\right|^{s} d x \quad \text { for each } s, 1 \leqq s \leqq p,
$$

(v) $\quad \sum_{k=1}^{\infty} \int_{-\pi}^{\pi}\left|w_{k}(x)\right|^{s} d x \leqq 2^{s+1} \int_{-\pi}^{\pi}\left|u^{\prime}(x)\right|^{s} d x \quad$ for each $s, 1 \leqq s \leqq p$,

(vi) there exists a sequence $\left\{I_{k}\right\}$ of disjoint intervals such that the support of $w_{k}$ is contained in $I_{k}$ and

$$
\begin{aligned}
\sum_{k=1}^{\infty}\left|I_{k}\right| \leqq \frac{1}{a^{r}} \int_{-\pi}^{\pi}\left|u^{\prime}(x)\right|^{r} d x, & \\
\int_{-\pi}^{\pi} w_{k}(x) d x=0, & k=1,2,3, \cdots .
\end{aligned}
$$

ProOF. We define $u$ by (ii) and put $u^{\prime}=f-u$. Now we decompose $u^{\prime}$. If we note that $a>a_{0}$, by the definition of $a_{0}$ we have

$$
\frac{\pi}{2}=\frac{1}{a_{0}^{r}} \int_{-\pi}^{\pi}|f(x)|^{r} d x \geqq \frac{1}{a^{r}} \int_{-\pi}^{\pi}\left|u^{\prime}(x)\right|^{r} d x .
$$

Let us divide the interval $(-\pi, \pi)$ into the four intervals of same length. The mean value of $\left|u^{\prime}(x)\right|^{r}$ over every intervals is less than $a^{r}$ by (2.5). Divide each interval into two equal intervals and let $I_{11}, I_{12}, I_{13}, \ldots$ be those intervals over which the mean of $\left|u^{\prime}\right|^{r}$ is not smaller than $a^{r}$. We have

$$
a^{r}\left|I_{1 k}\right| \leqq \int_{I_{1 k}}\left|u^{\prime}(x)\right|^{r} d x \leqq 2 a^{r}\left|I_{1 k}\right| \text {. }
$$

For if $I_{1 k}$ is obtained by subdivision of $I^{\prime}$, by our construction 


$$
a^{r}\left|I_{1 k}\right| \leqq \int_{I_{1 k}}\left|u^{\prime}(x)\right|^{r} d x \leqq \int_{I^{\prime}}\left|u^{\prime}(x)\right|^{r} d x \leqq a^{r}\left|I^{\prime}\right|=2 a^{r}\left|I_{1 k}\right|
$$

We set

$$
\begin{array}{ll}
v(x)=\frac{1}{\left|I_{1 k}\right|} \int_{I} u^{\prime}(y) d y & \text { for } x \text { in } I_{1 k}, \\
w_{1 k}(x)= \begin{cases}u^{\prime}(x)-v(x) & \text { for } x \text { in } I_{1 k} \\
0 & \text { elswhere, }\end{cases}
\end{array}
$$

$k=1,2,3, \cdots$.

Next we make a new subdivision of the intervals which are not in $\left\{I_{1 k}\right\}$. Denoting by $I_{2 k}$ the intervals over which the mean value of $\left|u^{\prime}\right|$ is not smaller than $a^{r}$, we extend the definition (2.7) and (2.8) to those intervals. Continuiting in this way, we get the sequences of functions $w$ 's and intervals $I$ 's; for simplicity we write them by $\left\{w_{k}\right\}$ and $\left\{I_{k}\right\}$. If we write $O=\bigcup_{k=1}^{\infty} I_{k}$ and define

$$
v(x)=u^{\prime}(x) \quad \text { for } x \text { in }(-\pi, \pi)-O,
$$

then it is clear that (i), (vi) and (vii) hold. By Hölder's inequality with exponents $s$ and $s^{\prime}, 1 / s+1 / s^{\prime}=1$, we have

$|v(x)|^{s} \leqq \frac{1}{\left|I_{k}\right|^{s}}\left(\int_{I_{k}}\left|u^{\prime}(y)\right| d y\right)^{s} \leqq \frac{\left|I_{k}\right|^{s / s^{\prime}}}{\left|I_{k}\right|^{s}} \int_{I_{k}}\left|u^{\prime}(y)\right|^{s} d y=\frac{1}{\left|I_{k}\right|} \int_{I_{k}}\left|u^{\prime}(y)\right|^{s} d y$

for $x$ in $I_{k}$. Thus we have

$$
\begin{aligned}
\int_{-\pi}^{\pi}|v(x)|^{s} d x & =\left(\int_{C O}+\sum_{k=1}^{\infty} \int_{I_{k}}\right)|v(x)|^{s} d x \\
& \leqq \int_{C O}\left|u^{\prime}(x)\right|^{s} d x+\sum_{k=1}^{\infty} \int_{I_{k}}\left|u^{\prime}(y)\right|^{s} d y=\int_{-\pi}^{\pi}\left|u^{\prime}(y)\right|^{s} d y
\end{aligned}
$$

where $C O=(-\pi, \pi)-O$ and

$$
\begin{aligned}
\int_{-\pi}^{\pi}\left|w_{k}(x)\right|^{s} d x & \leqq 2^{s}\left\{\int_{I_{k}}\left|u^{\prime}(x)\right|^{s} d x+\int_{I_{k}}|v(x)|^{s} d x\right\} \\
& \leqq 2^{s}\left\{\int_{I_{k}}\left|u^{\prime}(x)\right|^{s} d x+\int_{I_{k}}\left|u^{\prime}(y)\right|^{s} d y\right\}=2^{s+1} \int_{I_{k}}\left|u^{\prime}(y)\right|^{s} d y
\end{aligned}
$$

using (2.8) again. Therefore (iv) and (v) are proved. To prove (iii) we use (2.8) with $s=r$. If $x$ belongs to some interval $I_{k}$, we have

$$
|\vartheta(x)|^{r} \leqq \frac{1}{\left|I_{k}\right|} \int_{I_{k}}\left|u^{\prime}(y)\right|^{r} d y \leqq 2 a^{r}
$$


by (2.6). On the other hand if $x$ does not belong to any intervals $I_{k}$, there are arbitrarily small intervals containing $x$ such that

$$
\frac{1}{|I|} \int_{I}\left|u^{\prime}(x)\right|^{r} d x \leqq a^{r},
$$

by our choice of $I_{k}$. Hence $|v(x)|=\left|u^{\prime}(x)\right| \leqq a$ for a.e. $x$ in $C O$.

Therefore our proof is completed.

LEMMA 2. With the notations of (2.3) and Lemma 1, we have

$$
\sum_{k=1}^{\infty} \int_{C E}\left|K w_{k}\right| d x \leqq A \int_{-\pi}^{\pi}\left|u^{\prime}\right| d x,
$$

where $E$ is the set obtained by expanding each $I_{k}$ concentrically three times and $C E=(-\pi, \pi)-E$ and $A$ is some constant ${ }^{1)}$ not depending on $f$ and $a>a_{0}$.

PROOF. This lemma is known, but for the sake of completeness we show it.

Let us fix $k$ and put $I_{k}=I=(a-h, a+h)$ and $I_{k}^{*}=I^{*}=(a-3 h, a+$ 3h) $\cap(-\pi, \pi)$, then we have

$$
\int_{C E}\left|K w_{k}\right| d x \leqq \frac{1}{2} \int_{C I^{*}}\left|w_{k}\right| d x+\frac{1}{2} \int_{C I^{*}}\left|\widetilde{w}_{k}\right| d x
$$

Using (vii) in Lemma 1 , we have

$$
\begin{aligned}
& \int_{C I^{*}}\left|\widetilde{w}_{k}\right| \leqq \frac{1}{2 \pi} \int_{I^{*}} d x\left|\int_{-\pi}^{\pi}\left(\frac{1}{\tan (x-y) / 2}-\frac{1}{\tan (x-a) / 2}\right) w_{k}(y) d y\right| \\
& \leqq \frac{1}{2 \pi} \int_{-\pi}^{\pi}\left|w_{k}(y)\right| d y \int_{C I^{*}}\left|\frac{1}{\tan (x-y) / 2}-\frac{1}{\tan (x-a) / 2}\right| d x \\
& \leqq \frac{1}{2 \pi} \int_{-h}^{h}\left|w_{k}(y+a)\right| d y \int_{(-\pi, \pi)-(-3 h, 3 h)}\left|\frac{1}{\tan (x-y) / 2}-\frac{1}{\tan x / 2}\right| d x \\
& \leqq \frac{1}{2 \pi} \int_{-h}^{h}\left|w_{k}(y+a)\right| d y \int_{(-\pi, \pi)-(-3 h, 3 h)}\left|\frac{\sin y / 2}{(\sin (x-y) / 2) \sin x / 2}\right| d x .
\end{aligned}
$$

Noting that $0<h \leqq \pi / 2$ by our construction, it is easily verified that the inner integral does not exceed

$$
A^{\prime} \int_{3 h}^{\infty}\left|\frac{h}{(x-y) x}\right| d x<A^{\prime \prime},|y| \leqq h .
$$

Hence we get $\int_{C I^{*}}\left|\widetilde{w}_{k}\right| d x \leqq\left(A^{\prime \prime} / 2\right) \int_{I}\left|w_{k}\right| d x$ and

1) $A, A^{\prime}, A^{\prime \prime}$, are some constants and may be different in each occasion. 


$$
\int_{C E}\left|K w_{k}\right| d x \leqq\left(1 / 2+A^{\prime \prime} / 2\right) \int_{-\pi}^{\pi}\left|w_{k}\right| d x .
$$

Summing up over $k$ and using (v) of Lemma 1 with $s=1$, we get

$$
\begin{aligned}
\sum_{k=1}^{\infty} \int_{C E}\left|K w_{k}\right| d x & \leqq\left(1 / 2+A^{\prime \prime} / 2\right) \sum_{k=1}^{\infty} \int_{-\pi}^{\pi}\left|w_{k}\right| d x \\
& \leqq\left(1+A^{\prime \prime}\right) \int_{-\pi}^{\pi}\left|u^{\prime}\right| d x,
\end{aligned}
$$

which prove the lemma.

3. Main Theorem. An operator $T$ which maps into the scalar valued functions, is called quasi-linear if $T(f+g)$ is uniquely defined whenever $T f$ and $T g$ are defined, and if

$$
|T(f+g)| \leqq \kappa(|T f|+|T g|),
$$

where $\kappa$ is a constant independent on $f$ and $g$.

THEOREM 1. Suppose that a quasi-linear operator $T$ of $H_{p_{i}}$ to $\mu$-measurable functions satisfies

$$
\mu(\{s:|(T \varphi)(s)|>t\})^{1 / q_{i}} \leqq \frac{M_{i}}{t}\|\varphi\|_{p_{i}}
$$

for all $\phi$ in $H_{p_{i}}(i=0,1)$ and for all $t>0$, where $1 \leqq p_{i} \leqq q_{i}<\infty(i=0,1)$, $p_{0} \neq p_{1}$ and $q_{0} \neq q_{1}$. Let us put

$1 / p=(1-\theta) / p_{0}+\theta / p_{1}$ and $1 / q=(1-\theta) / q_{0}+\theta / q_{1} \quad(0<\theta<1)$.

Then $T$ can be extended to an operator on $H_{p}$ and satisfies

$$
\|T K f\|_{q} \leqq A(\kappa+1)^{2} M_{0}^{1-\theta} M_{1}^{\theta}\|f\|_{p}
$$

for all $f$ in $L_{p}(-\pi, \pi)$, where $A$ depends only on $p_{0}, p_{1}, q_{0}, q_{1}$ and $\theta$, and

$$
A^{q}=O\left[(p-1)^{-q_{0}}\left(q-q_{0}\right)^{-1}+\left(q_{1}-q\right)^{-1}\right] .
$$

PROOF. First we give the following remarks (cf. [6; vol.2, p.112]). If $f$ is a non-negative $\mu$-measurable function and $p \geqq 1$, we have

$$
\int f^{p} d \mu=-\int_{0}^{\infty} y^{p} d \mu(\{s: f(s)>y\})=p \int_{0}^{\infty} y^{p-1} \mu(\{s: f(s)>y\}) d y
$$

by the definition of Lebesgue-Stieltjes integral and integration by parts. Therefore if we denote $\mu(\{s:|f(s)|>y\})$ by $\mu(|f|>y)$, then

$$
\|T K f\|_{q}^{q}=q \int_{0}^{\infty} y^{q-1} \mu(|T K f|>y) d y .
$$


Next as is easily verified, we have

$$
\begin{gathered}
\mu(|T(f+g+h)|>3 \kappa(\kappa+1) y) \\
\leqq \mu(|T f|>y)+\mu(|T g|>y)+\mu(|T h|>y),
\end{gathered}
$$

since $|T(f+g+h)| \leqq \kappa(\kappa+1)(|T f|+|T g|+|T h|)$.

From now we use the notations of Lemma 1 : we put $r=(p+1) / 2$.

We consider separately the four cases.

Case (i ). $1=p_{0}<p_{1}, q_{0}<q_{1}$. Let $f \in L_{p}(-\pi, \pi)$, then by (3.5) we have

$$
\begin{aligned}
\|T K f\|_{q}^{q} & =q\left(\int_{0}^{3 \kappa(\kappa+1) y_{0}}+\int_{3 \kappa(\kappa+1) y_{0}}^{\infty}\right) y^{q-1} \mu(|T K f|>y) d y \\
& =q \int_{0}^{3 \kappa(\kappa+1) y_{0}} t^{q-1} \mu(|T K f|>t) d t \\
& +q(3 \kappa(\kappa+1))^{q} \int_{y_{0}}^{\infty} y^{q-1} \mu(|T K f|>3 \kappa(\kappa+1) y) d y .
\end{aligned}
$$

We put

$$
a=(y / B)^{\lambda} \text { and } a_{0}=\left(y_{0} / B\right)^{\lambda},
$$

where $\lambda$ and $B$ are constants determined later. We assume $\lambda>0$. Since $a>a_{0}$ if $y>y_{0}$, we can decompose $f$ into $u, v$ and $w$ by Lemma 1 .

Hence using (3.6) we have

$$
\begin{gathered}
\|T K f\|_{q}^{q} \leqq q \int_{0}^{3 \kappa(\kappa+1) y_{0}} t^{q-1} \mu(|T K f|>t) d t \\
+q(3 \kappa(\kappa+1))^{q}\left\{\int_{y_{0}}^{\infty} y^{q-1} \mu(|T K u|>y) d y+\int_{y_{0}}^{\infty} y^{q-1} \mu(|T K v|>y) d y\right. \\
\left.\quad+\int_{y_{0}}^{\infty} y^{q-1} \mu(|T K w|>y) d y\right\} \\
=q I_{1}+q(3 \kappa(\kappa+1))^{q}\left\{I_{2}+I_{3}+I_{4}\right\}, \text { say. }
\end{gathered}
$$

Now we estimate $I_{1}, I_{2}, I_{3}$ and $I_{4}$.

By the definition of $y_{0}$ and Hölder's inequality with exponents $p / r$ and $p^{\prime}(p-r)$, we have

$$
y_{0}=B a_{0}^{1 / \lambda}=B\left(\frac{2}{\pi} \int_{-\pi}^{\pi}|f|^{r} d x\right)^{1 / r \lambda} \leqq B\left(\frac{4^{p / r}}{2 \pi} \int_{-\pi}^{\pi}|f|^{p} d x\right)^{1 / p}
$$

By (3.2), we have

$$
\begin{aligned}
& I_{1} \leqq M_{0}^{q_{0}} \int_{0}^{3 \kappa(\kappa+1) y_{0}} t^{q-q_{0}-1}\|K f\|_{p_{0}}^{q_{0}} d t \\
& \leqq M_{0}^{q_{0}}\|K f\|_{p_{0}}^{q_{0}} \int_{0}^{3 \kappa(\kappa+1) y_{0}} t^{q-q_{r}-1} d t .
\end{aligned}
$$


AN EXTENSION OF THE INTERPOLATION THEOREM

349

By Hölder's inequality and M.Riesz' inequality $\|K f\|_{p} \leqq A_{p}\|f\|_{p}, \quad A_{p}=$ $O\left\{(p-1)^{-1}\right\}, p>1$, the last expression does not exceed

$$
\begin{aligned}
& M_{0}^{q_{0}}\|K f\|_{p}^{q_{0}}\left\{3 \kappa(\kappa+1) y_{0}\right\}^{q-q_{0}} /\left(q-q_{0}\right) \\
\leqq & M_{0}^{q_{0}} A_{p}^{q_{0}}\|K f\|_{p}^{q_{0}}\left\{3(\kappa+1)^{2}\right\}^{q} y_{0}^{q_{0}-q_{0}} /\left(q-q_{0}\right)
\end{aligned}
$$

Hence we get

$$
I_{1} \leqq\left(\frac{4^{p / r}}{2 \pi}\right)^{\left(q-q_{0}\right) / \lambda_{p}}\left\{3(\kappa+1)^{2}\right\}^{q} \frac{M_{0}^{q_{0}} A_{p}^{q_{0}} B^{q-q_{0}}}{q-q_{0}}\|f\|_{p}^{q_{0}+\left(q-q_{0}\right) / \lambda}
$$

By (3.2) and M.Riesz' inequality, we get

$$
\begin{aligned}
& I_{2} \leqq M_{1}^{q_{1}} \int_{y_{0}}^{\infty} y^{q-q_{1}-1}\|K u\|_{p_{1}}^{q_{1}} d y \\
& \leqq M_{1}^{q_{1}} A_{p_{1}}^{q_{1}} \int_{y_{0}}^{\infty} y^{q-q_{1}-1}\left\{\int|u(x)|^{p_{1}} d x\right\}^{q_{1} / p_{1}} d y .
\end{aligned}
$$

Applying Minkowski's inequality $\left\{\int_{F}\left|\int_{E} h(x, y) d x\right|^{r} d y\right\}^{1 / r} \leqq \int_{E}\left\{\int_{F}|h(x, y)|^{r} d y\right\}^{1 / r} d x$ $(r \geqq 1)$, the last integral does not exceed

$$
\begin{aligned}
& {\left[\int d x\left\{\int_{y_{0}}^{\infty} y^{q-q_{1}-1}|u(x)|^{q_{1}} d y\right\}^{p_{1} / q_{1}}\right]^{q_{1} / p_{1}} } \\
\leqq & {\left[\int d x\left\{\int_{B|y|}^{\infty} y^{q / \lambda} y^{q-q_{1}-1}|f|^{q_{1}} d y\right\}^{p_{1} / q_{1}}\right]^{q_{1} / p_{1}} } \\
= & \frac{B^{q-q_{1}}}{q_{1}-q}\left\{\int|f|^{p_{1}+\left[\left(q-q_{1}\right) p_{1} / q_{1} \lambda\right]} d x\right\}^{q_{1} / p_{1}}
\end{aligned}
$$

Using (3.2) and M.Riesz' inequality again we have

$$
I_{3} \leqq M_{1}^{q_{1}} A_{1}^{q_{1}} \int_{q_{0}}^{\infty} y^{q-q_{1}-1}\left\{\int|v(x)|^{p_{1}} d x\right\}^{q_{1} / p_{1}} d y .
$$

Since $|v(x)|^{p_{1}} \leqq 2^{\left(p_{1}-1\right) / r} B^{-\lambda\left(p_{1}-1\right)} y^{\lambda\left(p_{1}-1\right)}|v(x)|$ by (iii) of Lemma 1 , the last integral is bounded by

$$
2^{q_{1}\left(p_{1}-1\right) / 2 p_{1}} B^{-\lambda\left(p_{1}-1\right) q_{1} / p_{1}} \int_{y_{0}}^{\infty} y^{q-q_{1}-1+\left[\left(p_{1}-1\right) q_{1} \lambda / p_{1}\right]}\left\{\int|v(x)| d x\right\}^{q_{1} / p_{1}} d y .
$$

Applying (iv) of Lemma 1 with $s=1$ and Minkowski's inequality, the above integral does not exceed

$$
\begin{aligned}
& \int_{y_{0}}^{\infty} y^{q-q_{1}-1+\left[\left(p_{1}-1\right) q_{1} \lambda / p_{1}\right]}\left\{\int\left|u^{\prime}(x)\right| d x\right\}^{q_{1} / p_{1}} d y \\
\leqq & {\left[\int d x\left\{\int_{v_{0}}^{\infty} y^{q-q_{1}-1+\left[\left(p_{1}-1\right) q_{1} \lambda / p_{1}\right]}\left|u^{\prime}(x)\right|^{q_{1} / p_{1}} d y\right\}^{p_{1} / q_{1}}\right]^{q_{1} / p_{1}} }
\end{aligned}
$$


350

$$
\begin{aligned}
& \leqq\left[\int d x\left\{\int_{0}^{B|f|^{1 / \lambda}} y^{q-q_{1}-1+\left[\left(p_{1}-1\right) q_{1} \lambda / p_{1}\right]}|f|^{q_{1} / p_{1}} d y\right\}^{p_{1} / q_{1}}\right]^{q_{1} / p_{1}} \\
& =\frac{B^{q-q_{1}+\left[\left(q-q_{1}\right) p_{1} / \lambda q_{1}\right]}}{q-q_{1}+\left[\left(p_{1}-1\right) q_{1} \lambda / p_{1}\right]}\left\{\int|f|^{\left[\left(q-q_{1}\right) p_{1} / \lambda q_{1}\right]+p_{1}} d x\right\}^{q_{1} / p_{1}}
\end{aligned}
$$

Hence we get

$$
I_{3} \leqq 2^{q_{1}\left(p_{1}-1\right) / r p_{1}} \frac{M_{1}^{q_{1}} A_{p_{1}}^{q_{1}} B^{q-q_{1}}}{q-q_{1}+\left[\left(p_{1}-1\right) q_{1} \lambda / p_{1}\right]}\left\{\int|f|^{\left[\left(q-q_{1}\right) p_{1} / \lambda q_{1}\right]+p_{1}} d x\right\}^{q_{1} / p_{1}}
$$

By our hypotheses (3.2) and $p_{0}=1$, we have

$$
\begin{aligned}
I_{4} & \leqq M_{0}^{q_{0}} \int_{y_{0}}^{\infty} y^{q-q_{0}-1}\|K w\|_{p_{0}}^{q_{0}} d y \\
& \leqq 2^{q_{0}} M_{0}^{q_{0}}\left\{\int_{y_{0}}^{\infty} y^{q-q_{0}-1}\left(\int_{E}|K w| d x\right)^{q_{0}} d y^{\prime}+\int_{y_{0}}^{\infty} y^{q-q_{0}-1}\left(\int_{C E}|K w| d x\right)^{q_{0}} d y\right\} \\
& =2^{q_{0}} M_{0}^{q_{0}}\left(J_{1}+J_{2}\right),
\end{aligned}
$$

say, where $E$ is the set defined in Lemma 2 .

For $J_{2}$, we have

$$
\begin{aligned}
J_{2} & \leqq A^{q_{0}} \int_{y_{0}}^{\infty} y^{q-q_{0}-1}\left\{\int\left|u^{\prime}(x)\right| d x\right\}^{q_{0}} d y \\
& \leqq A^{q_{0}}\left[\int d x\left\{\int_{y}^{\infty} y^{q-q_{0}-1}\left|u^{\prime}(x)\right|^{q_{0}} d y\right\}^{1 / q_{0}}\right]^{q_{0}} \\
& \leqq A^{q_{0}}\left[\int d x\left\{\int_{0}^{B|\delta|^{1 / \lambda}} y^{q-q_{0}-1}|f(x)|^{q_{0}} d y\right\}^{1 / q_{0}}\right]^{q_{0}} \\
& =\frac{A^{q_{0}} B^{q-q_{0}}}{q-q_{0}}\left\{\int|f|^{\left[\left(q-q_{0}\right) / q_{\left.q_{0}\right]+1}\right.} d x\right\}^{q_{0}},
\end{aligned}
$$

using Lemma 2 for the first inequality and Minkowski's inequality for the second. On the other hand, by Hölder's inequality, we get

$$
J_{1} \leqq \int_{y}^{\infty} y^{q-q_{0}-1}|E|^{q_{0} / r^{\prime}}\left\{\int|K w|^{r} d x\right\}^{q_{0} / r} d y
$$

where $1 / r+1 / r^{\prime}=1$. Applying (vi) of Lemma 1 for $|E|$ and M.Riesz' inequality for the inner integral, we have

$$
J_{1} \leqq 3^{q_{0} / r^{\prime}} A_{r}^{q_{0}} B^{\lambda q_{s} r / r^{\prime}} \int_{y_{1}}^{\infty} y^{q-q_{0}-1-\left[q_{0} \lambda r / r^{\prime}\right]}\left\{\int\left|u^{\prime}\right|^{r} d x\right\}^{y_{0} / r^{\prime}}\left\{\int|w|^{r} d x\right\}^{q_{\mathrm{c}} / r} d y
$$

By (iv) of Lemma 1 with $s=r$, above integral is not greater than

$$
2^{(r+1) q_{0}} \int_{y_{0}}^{\infty} y^{q-q_{0}-1-\left[q_{0} \lambda r / r^{\prime}\right]}\left\{\int\left|u^{\prime}\right|^{r} d x\right\}^{q_{0}} d y .
$$


If we assume that

$$
q-q_{0}-\left[\lambda q_{0} r / r^{\prime}\right]>0,
$$

then by the same way as in estimating $J_{2}$, the integral does not exceed

$$
\begin{aligned}
& {\left[\int d x\left\{\int_{y_{0}}^{\infty} y^{q-q_{0}-1-\left[q_{0} \lambda r / r^{\prime}\right]}\left|u^{\prime}(x)\right|^{q_{0} r} d y\right\}^{q_{0}}\right]^{1 / q_{0}} } \\
\leqq & {\left[\int d x\left\{\int_{0}^{B|f|^{1 / \lambda}} y^{q-q_{0}-1-\left[q_{0} \lambda r / r^{\prime}\right]}\left|u^{\prime}(x)\right|^{q_{0} r} d y\right\}^{q_{0}}\right]^{1 / q_{0}} } \\
= & \frac{B^{q-q_{0}-\left[\lambda q_{0} r / r^{\prime}\right]}}{q-q_{0}-\left[\lambda q_{0} r / r^{\prime}\right]}\left\{\int|f|^{\left[\left(q-q_{0}\right) / q_{0} \lambda\right]+1} d x\right\}^{q_{0}} .
\end{aligned}
$$

Hence we get

$$
J_{1} \leqq 3^{q_{0} / r^{\prime}} 2^{(r+1) q_{0}} \frac{A_{r}^{q_{0}} B^{\left(q-q_{J}\right)}}{q-q_{0}-\left[\lambda q_{0} r / r^{\prime}\right]}\left\{\int|f|^{\left[\left(q-q_{0}\right) / q_{0} \lambda\right]+1} d x\right\}^{q_{0}}
$$

Therefore we have

$I_{4} \leqq 2^{q_{0}} M_{0}^{q_{o}}\left(\frac{3^{q_{0} / r^{\prime}} 2^{(r+1) q_{0} / r} A_{r}^{q_{0}} B^{\left(q-q_{0}\right)}}{q-q_{0}-\left[\lambda q_{0} r / r^{\prime}\right]}+\frac{A_{r}^{q_{0}} B^{q-q_{\mathrm{c}}}}{q-q_{0}}\right)\left(\int|f|^{\left.\left(q-q_{0}\right) / \lambda q_{0}\right]+}{ }_{1} d x\right)^{q_{0}}$.

Now if we put

$$
\lambda=\frac{p_{0}\left(q-q_{0}\right)}{q_{0}\left(p-p_{0}\right)}=\frac{p_{1}\left(q-q_{1}\right)}{q_{1}\left(p-p_{1}\right)},
$$

$q-q_{0}-\left[\lambda q r / r^{\prime}\right]=\left(q-q_{0}\right) / 2>0$ which is the assumption (3.14) and $|f|$ in $I_{1}, I_{2}, I_{3}$ and $I_{4}$ contain the same power $p$. Next we set

$$
B=M_{0}^{\rho} M_{1}^{\sigma}\|f\|_{p}^{\tau}
$$

and select $\rho, \sigma$ and $\tau$ so that the powers of $M_{0}, M_{1}$ and $\|f\|_{p}$ in $I$ 's are same, that is, we put

$$
\rho=-q_{0} /\left(q_{1}-q_{0}\right), \sigma=-q_{1} /\left(q_{0}-q_{1}\right) \text { and } \tau=\left(p_{1} q-p q_{1}\right) / p_{1}\left(q-q_{1}\right) .
$$

Then we obtain by (3.9), (3.10), (3.11) and (3.15)

$$
\|T K f\|_{q}^{q} \leqq(\kappa+1)^{2 q} A^{\prime} M_{o}^{(1-\theta) q} M_{1}^{\theta q}\left(\frac{1}{q-q_{0}}+\frac{1}{q_{1}-q}+\frac{1}{p-1}+\frac{1}{\left(q-q_{0}\right)(p-1)^{q_{0}}}\right)\|f\|_{p}^{q},
$$

where we used that $A_{r}, A_{p}=O(p-1)$ and $A^{\prime}$ is a constant depending only on $p_{0}, p_{1}, q_{0}$ and $q_{1}$.

Therefore we get Theorem in this case.

Case (ii). $1=p_{0}<p_{1}, q_{1}<q_{0}$. We can prove this case by the same way. Since $\lambda<0$ in this case, $a>a_{0}$ if $y<y_{0}$, therefore we put for $f$ in $L_{p}(-\pi, \pi)$ 


$$
\begin{gathered}
\|T K f\|_{q}^{q}=q\left(\int_{3 \kappa(\kappa+1) y_{0}}^{\infty}+\int_{0}^{3 \kappa(\kappa+1) y_{0}}\right) y^{q-1} \mu(|T K f|>y) d y \\
\leqq q \int_{3 \kappa(\kappa+1) y_{0}}^{\infty} t^{q-1} \mu(|T K f|>t) d t+q(3 \kappa(\kappa+1))^{q} \\
\left\{\int_{0}^{y_{0}} y^{q-1} \mu(|T K u|>y) d y+\int_{0}^{y_{0}} y^{q-1} \mu(|T K v|>y) d y+\int_{0}^{y_{0}} y^{q-1} \mu(|T K w|>y) d y\right\} \\
=q I_{1}+q(3 \kappa(\kappa+1))^{q}\left(I_{2}+I_{3}+I_{4}\right),
\end{gathered}
$$

say. $I_{1}, I_{2}, I_{3}$ and $I_{4}$ can be estimated by the same way as previous case but we must replace the domains of integrals $\left(0, B|f|^{1 / \lambda}\right)$, and $\left(B|f|^{1 / \lambda}, \infty\right)$ by $\left(B|f|^{1 / \lambda}\right.$, $\infty)$ and $\left(0, B|f|^{1 / \lambda}\right)$ respectively, and in the concluding expressions (3.9), (3.10), (3.11) and (3.15) the sign of the denominators will be changed. Further the inequality (3.8) will be replaced by

$$
y_{0} \geqq B\left(\frac{4^{p / r}}{2 \pi} \int|f|^{p} d x\right)^{1 / \lambda p}
$$

Case (iii). $1<p_{0}<p_{1}, q_{0}<q_{1}$. In this case our theorem degenerates to the special case of that of Marcinkiewicz's.

For every $a>0$, we put

$$
f=u+u^{\prime} \quad \text { and } \quad a=(y / B)^{\lambda},
$$

where $u=f$ if $|f|<a$ and $u=0$ otherwise, and $\lambda, B$ are the same constants as in case (i). (3.5) and similar inequality to (3.6) show that

$$
\begin{aligned}
\|T K f\|_{q}^{q} & =q \int_{0}^{\infty} y^{q-1} \mu(|T K f|>y) d y \\
& =(2 \kappa)^{q} q \int_{0}^{\infty} y^{q-1} \mu(|T K f|>2 \kappa y) d y \\
& \leqq(2 \kappa)^{q} q\left\{\int_{0}^{\infty} y^{q-1} \mu(|T K u|>y) d y+\int_{0}^{\infty} y^{q-1} \mu\left(\left|T K u^{\prime}\right|>y\right) d y\right\} \\
& =(2 \kappa)^{q} q\left(I_{1}+I_{2}\right),
\end{aligned}
$$

say. By the assumption (3.2) and M.Riesz' inequality, we have

$$
\begin{aligned}
I_{1} & \leqq M_{1}^{q_{1}} \int_{0}^{\infty} y^{q-q_{1}-1}\|K u\|_{p_{1}}^{q_{1}} d y \\
& \leqq A_{p_{1}}^{q_{1}} M_{1}^{q_{1}} \int_{0}^{\infty} y^{q-q_{1}-1}\|u\|_{p_{1}}^{q_{1}} d y .
\end{aligned}
$$

Remarking that $\lambda>0$ and arguing in the same way as (3.10), we get 


$$
\begin{aligned}
I_{1} & \leqq \frac{A_{p_{1}}^{q_{1}} M_{1}^{q_{1}} B^{q-q_{1}}}{q_{1}-q}\left\{\int|f|^{p_{1}+\left[\left(q-p_{1}\right) p_{1} / q_{1} \lambda\right]} d x\right\}^{q_{1} / p_{1}} \\
& =\frac{A_{p 1}^{q_{1}} M_{0}^{q(1-\theta)} M_{1}^{q \theta}}{q_{1}-q}\|f\|_{p}^{q} .
\end{aligned}
$$

For $I_{2}$ we have

$$
\begin{aligned}
I_{2} & \leqq M_{0}^{q_{0}} \int^{\infty} y^{q-q_{0}-1}\left\|K u^{\prime}\right\|_{p_{0}}^{q_{0}} d y \\
& \leqq M_{0}^{q_{0}} A_{p_{0}}^{q_{0}} \int_{0}^{\infty} y^{q-q_{0}-1}\left\|u^{\prime}\right\|_{p_{0}}^{q_{0}} d y \\
& \leqq M_{0}^{q_{0}} A_{p_{0}}^{q_{0}}\left[\int d x\left\{\int_{0}^{\infty} y^{q-q_{0}-1}\left|u^{\prime}(x)\right|^{q_{0}} d y\right\}^{p_{0} / q_{0}}\right]^{q_{0} / p_{0}} \\
& =M_{0}^{q_{0}} A_{p_{0}}^{q_{0}}\left[\int d x\left\{\int_{0}^{B||^{1 / \lambda}} y^{q-q_{0}-1}\left|u^{\prime}(x)\right|^{q_{0}} d y\right\}^{p_{0} / q_{0}}\right]^{q_{0} / p_{0}} \\
& =\frac{M_{0}^{q_{0}} A_{p_{0}}^{q_{0}} B^{q-q_{0}}}{q-q_{0}}\left\{\int|f|^{\left[\left(q-q_{0}\right) p_{0} / \lambda q_{0}\right]+1} d x\right\}^{q_{0} / p_{0}} \\
& =\frac{M_{0}^{(1-\theta) q} M_{1}^{\theta q}}{q-q_{0}}\|f\|_{p}^{q} .
\end{aligned}
$$

By (3.16), (3.17) and (3.18), we get the theorem.

Case (iv). $1<p_{0}<p_{1}, q_{1}<q_{0}$. We can prove this case by the same way as case (iii). But since $\lambda<0$, the integration domains $\left(0, B|f|^{1 / \lambda}\right)$ and $\left(B|f|^{1 / \lambda}, \infty\right)$ in (3.17) and (3.18) must be interchanged.

4. Several Variables Case. We consider the $n$-dimensional analogue of $\$ \S 2$ and 3 . We use the following notations; Euclidean space of $n$-dimension is denoted by $E_{n}$, its points $\left(x_{1}, x_{2}, \cdots, x_{n}\right),\left(y_{1}, y_{2}, \cdots, y_{n}\right)$, etc. by $X, Y$ etc. and the element of volume $d x_{1} d x_{2} \cdots d x_{n}$ by $d X$.

Now we define an analogue of $H_{p}$-class.

We put

$$
\widetilde{f}(X)=\lim _{\varepsilon \rightarrow 0} \int_{1 / \varepsilon \geqq|Y| \geqq \varepsilon} L(Y) f(X-Y) d Y,
$$

where $L(Y)$ is Calderón-Zygmund type kernel, that is, $L(Y)$ is locally integrable except the origin and satisfies the inequality

$$
\int_{|X|>A|\boldsymbol{X}|}|L(X-Y)-L(X)| d X \leqq A^{\prime}
$$

for all $Y$ in $E_{n}$ and there exists $q>1$ such that

$$
\|\widetilde{f}\|_{q} \leqq A^{\prime \prime}\|f\|_{q}
$$


for all $f$ in $L_{q}\left(E_{n}\right)$.

We define vector $\AA_{i} f$ by

$$
\Re f=(f, \tilde{f})
$$

and $\mathfrak{S}_{p}(p \geqq 1)$ as the space of all vectors such that $\Re f$ belongs to $L_{p}\left(E_{n}\right)$. Then we have

$$
\|\Re f\|_{p} \leqq A_{p}\|f\|_{p} \quad \text { for all } f \text { in } L_{p}\left(E_{n}\right),(4
$$

where $p>1$ and $A_{p}=O\left\{(p-1)^{-1}\right\}$ (cf.J.Schwartz [3]).

Specially the case $L(X)=X / c_{n}|X|^{n+1}$ was studied in detail by E.M.SteinG.Weiss [5], where $c_{n}=\pi^{(n+1) / 2} / \Gamma((n+1) / 2)$.

Next two lemmas correspond to Lemmas 1 and 2, respectively.

LEMMA 3. Let $f \in L_{p}\left(E_{n}\right)(\infty>p \geqq 1)$ and $p \geqq r \geqq 1$, then for each $a>0$ the following decomposition of $f$ is possible;

$$
f=u+u^{\prime}, u^{\prime}=v+w, w=\sum_{k=1}^{\infty} w_{k},
$$

$$
\begin{aligned}
& u=f, \text { if }|f|<a \text { and } u=0 \text { elswhere, } \\
& |v(X)| \leqq 2^{n / \boldsymbol{r}} a
\end{aligned}
$$

for a.e. $X$ in $E_{n}$,

$$
\int_{E_{n}}|v(X)|^{s} d X \leqq \int_{E_{n}}\left|u^{\prime}(X)\right|^{s} d X \quad \text { for each } s, 1 \leqq s \leqq p
$$

$$
\sum_{k=1}^{\infty} \int_{E_{n}}\left|w_{k}(X)\right|^{s} d X \leqq 2^{s+1} \int_{E_{n}}\left|u^{\prime}(X)\right|^{s} d X \quad \text { for each } s, 1 \leqq s \leqq p,
$$

(vi) there exists a sequence $\left\{I_{k}\right\}$ of disjoint cubes such that the support of $w_{k}$ is contained in $I_{k}$ and

$$
\begin{aligned}
\sum_{k=1}^{\infty}\left|I_{k}\right| \leqq \frac{1}{a^{r}} \int_{E_{n}}\left|u^{\prime}(X)\right|^{r} d X, & \\
\int_{E_{n}} w_{k}(X) d X=0, & k=1,2,3, \cdots .
\end{aligned}
$$

Proof. We define $u$ by (ii) and put $u^{\prime}=f-u$. Divide the space $E_{n}$ into a mesh of cubes of volume $>a^{-r} \int_{E_{n}}\left|u^{\prime}\right|^{r} d X$. Next divide each cube into $2^{n}$ equal cubes and let $I_{11}, I_{12}, I_{13}, \cdots$ be those cubes over which the mean value of $\left|u^{\prime}\right|^{r}$ is not smaller than $a^{r}$. Then we have the analogous inequality to (2.6) and the rest of arguments proceeds as in Lemma 1.

The following lemma will be proved by the same way as in Lemma 2, if we note the assumption (4.1). 
LEMMA 4. With the notations of Lemma 3, we have

$$
\sum_{k=1}^{\infty} \int_{C^{\prime} E}\left|\Re_{w_{k}}\right| d X \leqq A \int_{E_{\eta}}\left|u^{\prime}\right| d X,
$$

where $E$ is the set obtained by expanding each $I_{k}$ concentrically $A$ times and $C E=E_{n}-E$.

\section{5. n-dimensional Version of Theorem 1.}

THEOREM 2. Suppose that a quasi-linear operator $T$ of $\mathfrak{F}_{p_{i}}$ to $\mu$-measurable functions satisfies (3.1) and suppose that we have

$$
\mu\{s:|(T F)(s)|>t\}^{1 / q_{i}} \leqq \frac{M_{i}}{t}\|F\|_{p_{i}}
$$

for all $F$ in $\mathfrak{S}_{p_{i}}(i=0,1)$ and $t>0$, where $1 \leqq p_{i} \leqq q_{i}<\infty(i=0,1), p_{0} \neq p_{1}$, $q_{0} \neq q_{1}$. Let us define $p$ and $q$ by (3.1).

Then $T$ can be extended to an operator on $L_{p}\left(E_{n}\right)$ and satisfies

$$
\|T \hat{\Re} f\|_{q} \leqq A \kappa(\kappa+1) M_{0}^{1-\theta} M_{1}^{\theta}\|f\|_{p}
$$

for all $f$ in $L_{p}\left(E_{n}\right)$, where $A$ is a constant independent on $f$ and of the order of (3.4).

PROOF. Our proof proceeds as Theorem 1. We consider separately four cases too:
(i) $1=p_{0}<p_{1}, q_{0}<q_{1}$,
(ii) $1=p_{0}<p_{1}, q_{1}<q_{0}$,
(iii) $1<p_{0}<p_{1}, q_{0}<q_{1}$,
(iv) $1<p_{0}<p_{1}, q_{1}<q_{0}$.

But since the proofs of Cases (iii) and (iv) are not different from the corresponding cases of Theorem 1, we treat only Cases (i) and (ii).

We use the notations of Lemmas 3 and 4 .

Case (i). $1=p_{0}<p_{1}, q_{0}<q_{1}$. Let $f \in L_{p}\left(E_{n}\right)$. Using (3.5) replaced by and (3.6), we have

$$
\begin{aligned}
\|T \Re f\|_{q}^{q}= & q \int_{0}^{\infty} y^{q-1} \mu(|T \Re f|>y) d y \\
\leqq & q(3 \kappa(\kappa+1))^{q}\left\{\int_{0}^{\infty} y^{q-1} \mu(|T \Re u|>y) d y+\right. \\
& \left.\int_{0}^{\infty} y^{q-1} \mu(|T \hat{\Re}|>y) d y+\int_{0}^{\infty} y^{q-1} \mu(|T \Re w|>y) d y\right\} \\
= & q(3 \kappa(\kappa+1))^{q}\left(I_{1}+I_{2}+I_{3}\right),
\end{aligned}
$$

say. We put $a=(y / B)^{\lambda}$ where $\lambda$ and $B$ are same as before.

For $I_{1}$ using the arguments of $I_{2}$ of Case (i) in Theorem 1 but applying Calderón-Zygmund inequality (4.2), we have 


$$
\begin{aligned}
I_{1} & \leqq M_{1}^{q_{1}} \int_{0}^{\infty} y^{q-q_{1}-1}\|\Re u\|_{p_{1}}^{q_{1}} d y \\
& \leqq M_{1}^{q_{1}} A_{p_{1}}^{q_{1}} \int_{0}^{\infty} y^{q-q_{1}-1}\left\{\int|u(X)|^{p_{1}} d X\right\}^{q_{1} / p_{1}} d y \\
& \leqq M_{1}^{q_{1}} A_{p_{1}}^{q_{1}}\left[\int d X\left\{\int_{0}^{\infty} y^{q-q_{1}-1}|u|^{q_{1}} d y\right\}^{p_{1} / q_{1}}\right]^{q_{1} / p} \\
& =M_{1}^{q_{1}} A_{p_{1}}^{q_{1}}\left[\int d X\left\{\int_{B|\rho|^{1 / \lambda}}^{\infty} y^{q-q_{1}-1}|f|^{q_{1}} d y\right\}^{p_{1} / q_{1}}\right]^{q_{1} / p_{1}} \\
& =\frac{M_{1}^{q_{1}} A_{p_{1}}^{q_{1}} B^{q-q_{1}}}{q_{1}-q}\left\{\int|f|^{p_{1}+\left[\left(q-q_{1}\right) p_{1} / q_{1} \lambda\right]} d X\right\}^{q_{1} / p_{1}} \\
& =\frac{A_{p_{1}}^{q_{1}} M_{0}^{(1-\theta) q} M_{1}^{q q}}{q_{1}-q}\|f\|_{p}^{q} .
\end{aligned}
$$

By (5.1), (4.2) and (iii) of Lemma 3, we get

$$
\begin{aligned}
& I_{2} \leqq M_{1}^{q_{1}} A_{p_{1}}^{q_{1}} \int_{0}^{\infty} y^{q-q_{1}-1}\left\{\int|v(X)|^{p_{1}} d X\right\}^{q_{1} / p_{1}} d y \\
& \leqq M_{1}^{q_{1}} A_{p_{1}}^{q_{1}} 2^{n\left(p_{1}-1\right) q_{1} / r p_{1}} B^{-\lambda\left(p_{1}-1\right) q_{1} / p} \int_{0}^{\infty} y^{q-q_{1}-1+\left[\left(p_{1}-1\right) q_{1} \lambda / p_{1}\right]}\left\{\int|v(X)| d X\right\}^{q_{1} / p_{1}} d y \\
& \leqq M_{1}^{q_{1}} A_{p_{1}}^{q_{1}} 2^{n\left(p_{1}-1\right) q_{1} / p_{1}} B^{-\lambda\left(p_{1}-1\right) q_{1} / p_{1}}\left[\int d X\left\{\int_{0}^{\infty} y^{q-q_{1}-1+\left[\left(p_{1}-1\right) q \lambda / p_{1}\right]}\left|u^{\prime}(X)\right|^{q_{1} / p_{1}} d y\right\}^{p_{1} / q}\right]^{q_{1} / p_{1}} \\
& \leqq M_{1}^{q_{1}} A_{p_{1}}^{q_{1}} 2^{n\left(p_{1}-1\right) q_{1} / p_{1}} B^{-\lambda\left(p_{1}-1\right) q_{1} / p_{1}}\left[\int d X\left\{\int_{0}^{B|f|^{1 / \lambda}} y^{q-q_{1}-1+\left[\left(p_{1}-1\right) q \mid \lambda / p_{1}\right]}|f|^{q_{1} / p_{1}} d y\right\}^{p_{1} / q_{1}}\right]^{q_{1} / p_{1}} \\
& =\frac{M_{1}^{q_{1}} A_{p_{1}}^{q_{1}} 2^{n\left(p_{1}-1\right) q_{1} / p_{1}} B^{-\lambda\left(p_{1}-1\right) q_{1} / p_{1}}}{q-q_{1}+\left[\left(p_{1}-1\right) q_{1} \lambda / p_{1}\right]}\left\{\int|f|^{p_{1}+\left[\left(q-q_{1}\right) p_{1} / \lambda q_{1}\right]} d X\right\}^{q_{1} / p_{1}} \\
& =2^{n\left(p_{1}-1\right) q_{1} / r p_{1}} A_{\nu_{1}}^{q_{1}} M_{0}^{(1-\theta) q} M_{1}^{\theta q} \frac{\left(p_{1}-p\right)}{\left(q_{1}-q\right)(p-1)}\|f\|_{p}^{q} .
\end{aligned}
$$

For $I_{3}$ we put as (3.12)

$$
\begin{aligned}
& I_{3} \leqq M_{0}^{q_{0}} \int_{0}^{\infty} y^{q-q_{0}-1}\|\Re w\|_{p_{0}}^{q_{0}} d y \\
& \leqq 2^{q_{0}} M_{0}^{q_{0}}\left\{\int_{0}^{\infty} y^{q-q_{0}-1}\left(\int_{E}|\Re w| d X\right)^{q_{0}} d y+\int_{0}^{\infty} y^{q-q_{0}-1}\left(\int_{C_{E}}|\Re w| d X\right)^{q_{0}} d y\right\} \\
& =2^{q_{0}} M_{0}^{q_{0}}\left(J_{1}+J_{2}\right),
\end{aligned}
$$

say, where $E$ is the set defined in Lemma 4 . By the last lemma, we get

$$
J_{2} \leqq A^{q_{n}} \int_{0}^{\infty} y^{q-q_{0}-1}\left\{\int_{E_{n}}\left|u^{\prime}(X)\right| d X\right\}^{q_{0}} d y
$$




$$
\begin{aligned}
& \leqq A^{q_{0}}\left[\int d X\left\{\int_{0}^{\infty} y^{q-q_{0}-1}\left|u^{\prime}(X)\right|^{q_{0}} d y\right\}^{1 / q_{0}}\right]^{q_{0}} \\
& \leqq A^{q_{0}}\left[\int d X\left\{\int_{0}^{B / \rho^{1 / \lambda}} y^{q-q_{0}-1}|f(X)|^{q_{0}} d y\right\}^{1 / q_{0}}\right]^{q_{0}} \\
& =\frac{A^{q_{0}} B^{q-q_{0}}}{q-q_{0}}\left\{\int|f|^{\left[\left(q-q_{0}\right) / \lambda_{q_{0}}\right]+1} d X\right\} \\
& =\frac{A^{q_{0}} M_{0}^{(1-\theta) q} M_{1}^{\theta_{q}}}{q-q_{0}}\|f\|_{p}^{q} .
\end{aligned}
$$

By Hölder's inequality and (vi) of Lemma 3, we have

$$
\begin{aligned}
& J_{1} \leqq \int_{0}^{\infty} y^{q-q_{n}-1}|E|^{q_{0} / r^{\prime}}\left\{\int|\Re w|^{r} d X\right\}^{q_{0} / r} d y \\
& \leqq A^{q / r^{\prime}} A_{r}^{q_{0}} B^{\lambda q_{0} r / r^{\prime}} \int_{0}^{\infty} y^{q-q_{0}-1-\left[q_{0} \lambda r / r^{\prime}\right]}\left\{\int\left|u^{\prime}\right|^{r} d X\right\}^{q_{0} / r^{\prime}}\left\{\int|w|^{r} d X\right\}^{q_{0} / r} d y,
\end{aligned}
$$

where $1 / r+1 / r^{\prime}=1$. By virtue of (v) of Lemma 3 ,

$$
\begin{aligned}
& J_{1} \leqq A^{q_{0} / r^{\prime}} 2^{(r+1) q_{0} / r} A_{r}^{q_{0}} B^{\lambda q_{0} r / r^{\prime}} \int_{0}^{\infty} y^{q-q_{0}-1-\left[q_{0} \lambda r / r^{\prime}\right]}\left\{\int\left|u^{\prime}\right|{ }^{r} d X\right\}^{q_{0}} d y \\
& \leqq A^{q_{0} / r^{\prime}} 2^{(r+1) q_{0} / r} A_{r}^{q_{0}} B^{\lambda q_{0} r / r^{\prime}}\left[\int d X\left\{\int_{0}^{\infty} y^{q-q_{0}-1-\left[q_{0} \lambda r / r^{\prime}\right]}\left|u^{\prime}(X)\right|^{q_{0} r} d y\right\}^{q_{0}}\right]^{1 / q_{0}} \\
& \leqq A^{q_{0} / r^{\prime}} 2^{(r+1) q_{0} / r} A_{r}^{q_{0}} B^{\lambda q_{0} r / r^{\prime}}\left[\int d X\left\{\int_{0}^{B|f|^{1 / \lambda}} y^{q-q_{0}-1-\left[q_{0} \lambda r / r^{\prime}\right]}\left|u^{\prime}(X)\right|^{q_{0} r} d y\right\}^{q_{0}}\right]^{1 / q_{0}} \\
& \leqq \frac{A^{\prime} B^{q-q_{0}-\left[\lambda q_{0} r / r^{\prime}\right]}}{(r-1) q_{0}\left\{q-q_{0}-\left[\lambda q_{0} r / r^{\prime}\right]\right\}}\left\{\int|f|^{\left[\left(q-q_{0}\right) / q_{0} \lambda\right]+1} d X\right\}^{q_{0}} \\
& \leqq \frac{A^{\prime \prime}}{(p-1)^{q_{0}}\left(q-q_{0}\right)} M_{0}^{(1-\theta) q} M_{1}^{\theta q_{1}}\|f\|_{p}^{q},
\end{aligned}
$$

where $A^{\prime}$ and $A^{\prime \prime}$ depend only on $p_{0}, p_{1}, q_{0}$ and $q_{1}$.

Thus we obtain the estimation

$$
\|T \Re f\|_{q}^{q} \leqq \kappa^{q}(\kappa+1)^{q} A^{\prime \prime \prime} M_{0}^{(1-\theta) q} M_{1}^{\theta q}\left(\frac{1}{q_{1}-q}+\frac{1}{(p-1)^{q_{0}}\left(q-q_{0}\right)}\right)\|f\|_{p}^{q},
$$

$A^{\prime \prime \prime}$ depending only on $p_{i}$ and $q_{i}(i=0,1)$.

Therefore a proof of Case (i) is completed.

Case (ii). $1=p_{0}<p_{1}, q_{1}<q_{0}$. A proof of this case may proceed as above case. But the domains of integrals $\left(0, B|f|^{1 / \lambda}\right)$ and $\left(B|f|^{1 / \lambda}, \infty\right)$ in $I_{k}$ $(k=1,2,3)$ must be interchanged.

6. Some Applications. For the sake of convenience we restate the examples of application of our theorem. 
Littlewood-Paley function $g^{*}$ is defined by

$$
g^{*}(\theta, \varphi)=\left\{\sum_{n=1}^{\infty} \frac{\left|S_{n}(\theta)-\sigma_{n}(\theta)\right|^{2}}{n}\right\}^{1 / 2},
$$

where $S_{n}(\theta)$ and $\sigma_{n}(\theta)$ are $n$-th partial sum and $(C, 1)$-mean of Fourier series of $\phi$ in $H_{1}$ respectively. E.M.Stein [4] proved that this operator is weak type $(1,1)$ for $H_{1}$-class (on the other hand $g^{*}$ is not weak type $(1,1)$ for $L_{1}(-\pi, \pi)$, see [6, vol.II, p. 315]), and it is rather easily verified that $g^{*}$ is strong type $(2,2)$. Therefore we get the well-known inequality applying Theorem 1 ;

$$
\left\|g^{*}\right\|_{p} \leqq A_{p}\|f\|_{p} \quad \text { for all } f \text { in } L_{p}(-\pi, \pi) \text {, }
$$

where $A_{p}=O\left\{(p-1)^{-2}\right\}$ and $2 \geqq p>1$.

Another example is the operator $(T \varphi)(\theta)=S_{n(\theta)}(\theta)$, where $n(\theta)$ is any integral valued measurable function. This operator $T$ is strong type $(1,1)$ for $H_{1}$ and strong type (2.2) for $L_{2}(-\pi, \pi)$ (see [6, Chap. XIII]), when $d \mu\left(t^{\prime}\right)$ $=d \theta / \log (|n(\theta)|+2)$ with the notation of Theorem 1 . Since the factorization of functions in $H_{1}$ is possible by real methods (see e.g., W. Rudin; Fourier Analysis on Groups, Interscience, New York, 1962, p.205), our theorem gives a real proof of Littlewood-Paley inequality

$$
\int_{-\pi}^{\pi} \sup _{n \geqq 0}\left\{\frac{\left|S_{n}(x)\right|^{p}}{\log (n+2)}\right\} d x \leqq A_{p} \int_{-\pi}^{\pi}|f(x)|^{p} d x
$$

for all $f$ in $L_{p}(-\pi, \pi)(2 \geqq p>1)$, where $A_{p}$ is a constant.

CORRECTION: The estimation of the norm of $T$ of the Theorem in the previous paper, S. Igari [2] is incorrect, that is, $(p-1)^{-1}$ in $A^{q}$ must be replaced by $(p-1)^{-q_{0}}$.

\section{REFERENCES}

[1] L. HÖRMANDER, Estimates for translation invariant operators in $L_{p}$ spaces, Acta Math., 104(1960), 93-139.

[2] S. IGARI, An extension of the interpolation theorem of Marcinkiewicz, Proc. Japan Acad., 38(1962), 731-734.

[3] J.SCHWARTZ, A remark on inequality of Calderón-Zygmund type for vector-valued functions, Comm. Pure Appl. Math., 14(1961), 785-799.

[4] E. M.STEIN, On limit of operators, Ann. Math., 74(1961), 140-170.

[5] E.M.STEIN and G. WEISS, On the theory of harmonic functions of several variables.

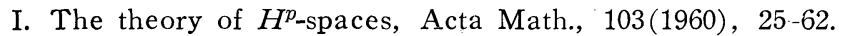

[6] A.ZYGmund, Trigonometric Series, 2nd edition, Cambridge (1959).

TÔHOKU UNIVERSITY. 